\title{
Pogitare
}

\section{DECLÍNIO DAS TAXAS DE INTERNAÇÃO HOSPITALAR POR DOENÇAS CARDIOVASCULARES EM ADULTOS NO BRASIL}

\author{
Fernanda Sabini Faix Figueiredo ${ }^{1}$ () \\ Thamires Fernandes Cardoso da Silva Rodrigues ${ }^{2}$ (1) \\ Luana Cristina Bellini Cardoso ${ }^{3}$ (i) \\ Fernanda Gatez Trevisan dos Santos ${ }^{3}$ (1) \\ Rosana Rosseto de Oliveira ${ }^{4}$ \\ Cremilde Aparecida Trindade Radovanovic ${ }^{3}$ (i)
}

\begin{abstract}
RESUMO
Objetivo: analisar a tendência das taxas de internação hospitalar por doenças cardiovasculares em adultos no Brasil.

Método:estudoecológico, de sériestemporais, dastaxas de internaçãopor doenças cardiovasculares em adultos por sexo, faixa etária e região de residência no Brasil no período de 2005 a 2016. Foram utilizados os dados do Sistema de Informações Hospitalares do Sistema Único de Saúde. Para as análises de tendência, utilizaram-se modelos de regressão polinomial.

Resultados: detectou-se tendência de declínio nas taxas de internação por doença cardiovascular em todo o país, sendo que a região Centro-Oeste obteve a redução mais expressiva (-21,29 ao ano). A região Sul deteve as maiores taxas de internação, inclusive por doenças isquêmicas do coração e cerebrovasculares.

Conclusão: o Brasil apresentou tendência decrescente nas taxas de internação, entretanto, ainda são elevadas. Os resultados podem repercutir nas ações de vigilância em saúde, especialmente em regiões cujas taxas de internação apresentam-se elevadas.
\end{abstract}

DESCRITORES: Doenças Cardiovasculares; Estudos de Séries Temporais; Hospitalização; Doença Crônica; Sistemas de Informação em Saúde.

\section{CAÍDA DE LAS TAJAS DE INTERNACION HOSPITALARIA POR ENFERMEDADES CARDIOVASCULARES EN ADULTOS EN BRASIL}

\section{RESUMEN:}

Objetivo: evaluar la tendencia de las tajas de internación hospitalaria por enfermedades cardiovasculares en adultos en Brasil. Método: estudio ecológico, de series temporales, de las tajas de internación por enfermedades cardiovasculares en adultos considerándose sexo, franja etaria y región de vivienda en Brasil en el periodo de 2005 a 2016. Se utilizaron los datos del Sistema de Informaciones Hospitalarias del Sistema Único de Salud. Para los análisis de tendencia, se emplearon modelos de regresión polinomial. Resultados: hubo tendencia de caída en las tajas de internación por enfermedad cardiovascular en todo el país; sin embargo la región Centro-Este obtuvo la reducción más expresiva (-21,29 al año). La región Sur presentó las mayores tajas de internación, incluso por enfermedades isquémicas del corazón y cerebrovasculares. Conclusión: Brasil presenta tendencia decreciente en las tajas de internación, pero todavía son altas. Los resultados pueden repercutir en las acciones de vigilancia en salud, en especial en regiones cuyas tajas de internación se muestran elevadas.

DESCRIPTORES: Enfermedades Cardiovasculares; Estudios de Series Temporales; Hospitalización; Enfermedad Crónica; Sistemas de Información en Salud. 
O desenvolvimento de doenças cardiovasculares (DCV) está diretamente associado a alguns fatores de risco, tais como idade, fatores genéticos, tabagismo, obesidade, sedentarismo, alimentação inadequada, consumo abusivo de bebida alcoólica, e presença de Hipertensão Arterial Sistêmica (HAS) ${ }^{(1)}$. Investir em ações de promoção e prevençã̃o para mudanças no estilo de vida se faz indispensável, uma vez que grande parte dos fatores de risco para o desenvolvimento de DCV é passível de modificação, e estudos brasileiros detectaram alta prevalência desses fatores na população jovem e adulta ${ }^{(2)}$.

Em todo o mundo, as DCV vêm diminuindo, entretanto, devido ao crescimento populacional, estilo de vida e ao envelhecimento, ainda se destacam no quadro de morbidade e mortalidade, especialmente as doenças isquêmicas do coração (DIC) e doenças cerebrovasculares (DCbV) ${ }^{(3)}$. Em Portugal, as DIC foram responsáveis por 4.688 mortes em 2014 e $2,4 \%$ de todos os anos perdidos por incapacidades ${ }^{(4)}$. Na Coreia do Sul, no ano de 2015, as DCV representaram a segunda causa de mortes do país, com 32.964 óbitos em homens e 37.848 em mulheres, uma taxa de mortalidade de $63,6 \%$ e $72,2 \%$ respectivamente ${ }^{(5)}$.

O Brasil se assemelha a esse cenário, visto que as DCV ocupam a quarta posição no ranking de causas de internações hospitalares. As DCV possuem encargos negativos que incidem sobre o indivíduo, família e sociedade, por resultar em sequelas que reduzem a qualidade de vida, elevam os gastos com cuidados de saúde, bem como por suscitar afastamento das atividades laborais ${ }^{(6)}$.

Neste estudo, buscou-se verificar a manifestação das DCV por meio das taxas de internação hospitalar em adultos. Sabe-se que tais agravos atingem proporções alarmantes, inclusive em adultos em idade produtiva; compreender o comportamento das DCV na população possibilita investigar as lacunas na atenção à saúde para o controle dessas doenças, e as internações são indicadores valiosos para entender o impacto delas para as regiões que necessitam de intervenções mais intensas. Podem produzir ainda subsídios para o planejamento das intervenções de enfermagem para o enfrentamento das DCV, da Atenção Primária à Saúde até a Atenção Hospitalar, com o intuito de buscar ações de educação em saúde, o vínculo com a população e a atenção integral à saúde dos indivíduos.

Neste cenário, reconhecendo a importância da prevenção de doenças que representam elevados índices de morbidade e mortalidade no país, e em virtude dos gastos elevados que estes agravos ocasionam ao sistema de saúde, este estudo teve por objetivo analisar a tendência das taxas de internação hospitalar por doenças cardiovasculares em adultos no Brasil.

\section{MÉTODO}

Trata-se de um estudo ecológico, de séries temporais com dados referentes às internações hospitalares por doenças cardiovasculares em adultos no Brasil. O Brasil é formado por 26 unidades federativas e o Distrito Federal, que estão distribuídos em cinco grandes regiões: Norte, Nordeste, Sudeste, Sul e Centro-Oeste. Para este estudo, o Distrito Federal foi analisado em conjunto com a região Centro-Oeste.

As informações são provenientes da Autorização de Internação Hospitalar (AlH), que possui informações sobre o diagnóstico e procedimentos realizados durante a hospitalização, a qual é processada e, a partir dela, emitem-se relatórios que são encaminhados ao Sistema de Informações Hospitalares do Sistema Único de Saúde (SIH/SUS) $)^{(7)}$. O SIH/SUS 
é uma ferramenta importante para a avaliação da qualidade das estratégias em saúde direcionadas à população, permitindo também a construção e acompanhamento do perfil de morbimortalidade hospitalar, direcionando ações de promoção e prevenção em saúde para uma população definida e acompanhamento do desempenho dos serviços hospitalares ${ }^{(8)}$.

Analisaram-se os registros de internações hospitalares por doenças do aparelho circulatório, disponíveis no Sistema de Informações Hospitalares do Sistema Único de Saúde (SIH/SUS), entre os anos de 2005 e 2016, de pessoas entre 20 e 59 anos. A escolha do período do estudo corroborou a implantação da Política Nacional de Promoção da Saúde, em 2006, cujo objetivo foi promover a qualidade de vida, reduzir a vulnerabilidade e os riscos à saúde (9). Optou-se então por estabelecer o ano de 2005 como o ponto inicial, seguindo até 2016, o último ano disponível na base de dados no momento inicial da coleta de dados, perfazendo um total de 12 anos.

Filtraram-se os dados por meio da lista de morbidades da Classificação Internacional de Doenças, em sua $10^{\text {a }}$ Revisão (CID-10), referente ao capítulo IX (Doenças Cardiovasculares - DCV, Doenças Isquêmicas do Coração - DIC e Doenças Cerebrovasculares - DCbV), selecionando-se as internações de adultos entre 20 e 59 anos (20-29, 30-39, 40-49 e 5059 anos), de ambos os sexos. A coleta ocorreu no mês de maio de 2017; inicialmente, os dados extraídos do SIH/SUS foram tabulados duplamente na planilha eletrônica Microsoft Office Excel 2007, em seguida realizou-se os procedimentos de análise estatística com auxílio do software Statistical Package for Social Sciences (SPSS), versão 20.0.

Para o cálculo das taxas de internação, realizou-se a razão entre o número de internações hospitalares por doenças cardiovasculares em adultos, pela população adulta correspondente ao mesmo período, multiplicado por 100.000 habitantes. As taxas de internação foram calculadas anualmente para os três grupos: total de DCV, DIC e DCbV.

A análise de tendência das taxas de internação se deu por meio da aplicação do modelo de regressão polinomial. Consideraram-se as taxas de internação como a variável dependente $(\mathrm{Y})$ e os anos do estudo como a variável independente ( $\mathrm{X}$ ). As séries temporais foram suavizadas por meio de média móvel de três pontos. Foram testados os modelos de regressão polinomial linear $(y=\beta 0+\beta 1 \times 1)$, quadrático $(y=\beta 0+\beta 1 \times 1+\beta 2 \times 2)$, e cúbico $(y=\beta 0+\beta 1 \times 1+\beta 2 \times 2+\beta 3 \times 3)$, quando necessário. Os modelos que apresentaram significância estatística, com melhor coeficiente de determinação e resíduo sem vício, foram considerados como melhor modelo polinomial. No caso de dois modelos apresentarem-se semelhantes, o de menor ordem foi selecionado, optando-se sempre pelo modelo mais simples. O nível de significância considerado foi de $5 \%$.

Este estudo recebeu a dispensa de análise ética pelo Comitê Permanente de Ética em Pesquisa Envolvendo Seres Humanos, sob parecer $n^{\circ} 18 / 2016$, visto que as informações foram obtidas em banco de dados de domínio público, sem a possibilidade de identificação individual.

\section{RESULTADOS}

Analisaram-se 5.710.607 internações por DCV, de adultos residentes no Brasil, no período de 2005 a 2016. Do total de internações, 20,27\% $(n=1.157 .540)$ tiveram como diagnóstico as DIC e 13,31\% $(n=760.082)$ as DُCbV. Notou-se que a taxa de internação por DCV no Brasil passou de 516,4 internações/100 mil habitantes em 2005, para 376,1 internações/100 mil habitantes em 2016.

A região Sul exibiu a maior taxa de internação por DCV $(670,6$ internações/100 mil habitantes em 2005 e 542,7 internações/100 mil habitantes em 2016), DCbV (85,3 
internações/100 mil habitantes em 2005 e 72,9 internações/100 mil habitantes em 2016) e DIC (153,5 internações/100 mil habitantes em 2005 e 159,3 internações/100 mil habitantes em 2016), sendo que esta última apresentou aumento médio de 3,77\%. Identificou-se que a região Centro-Oeste apresentou a maior redução média na taxa de internação por $\mathrm{DCV}$, passando de 666,6 internações/100 mil habitantes para 336,8 internações/100 mil habitantes, o que corresponde a uma redução de 49,47\% (Figura 1).

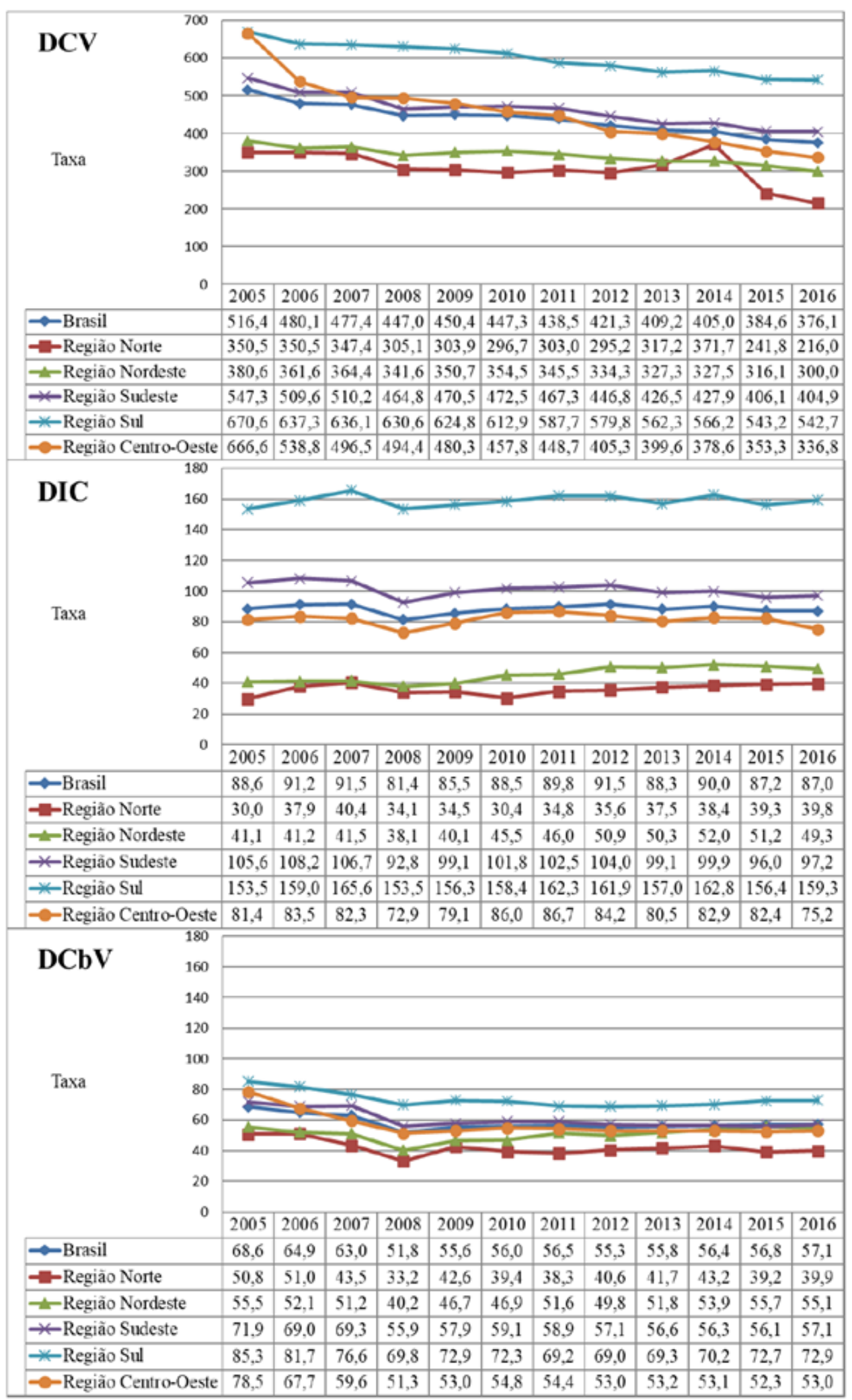

Figura 1 - Taxas de internação hospitalar por doenças cardiovasculares em adultos no Brasil, entre 2005 a 2016. Maringá, PR, Brasil, 2017 
$\mathrm{Na}$ análise de tendência, detectou-se declínio nas taxas de internação por DCV em todo o Brasil, com redução média de 10,37 ao ano $\left(r^{2}=0,98, p=<0,001\right)$. A região CentroOeste apresentou média ao ano de 21,29 $\left(r^{2}=0,98, p=<0,001\right)$, a redução mais expressiva entre as regiões brasileiras (Tabela 1).

Tabela 1 - Tendência das taxas de internação hospitalar por doenças cardiovasculares em adultos no Brasil entre 2005 a 2016. Maringá, PR, Brasil, 2017

Local

Modelo

R2

p Tendência*

Doenças Cardiovasculares

\begin{tabular}{lcccc}
\hline Brasil & $\mathrm{y}=436,01-10,37 \mathrm{x}$ & 0,98 & $<0,001$ & $\downarrow$ \\
\hline Região Norte & $\mathrm{y}=307,93-9,11 \mathrm{x}$ & 0,87 & $<0,001$ & $\downarrow$ \\
\hline Região Nordeste & $\mathrm{y}=345,07-5,29 \mathrm{x}$ & 0,95 & $<0,001$ & $\downarrow$ \\
\hline Região Sudeste & $\mathrm{y}=466,96-11,05 \mathrm{x}$ & 0,97 & $<0,001$ & $\downarrow$ \\
\hline Região Sul & $\mathrm{y}=604,87-11,37 \mathrm{x}$ & 0,99 & $<0,001$ & $\downarrow$ \\
\hline Região Centro-Oeste & $\mathrm{y}=459,68-21,29 \mathrm{x}$ & 0,98 & $<0,001$ & $\downarrow$ \\
\hline
\end{tabular}

Doenças Isquêmicas do Coração

\begin{tabular}{lcccc}
\hline Brasil & $y=87,42+1,16 x+0,16 x^{2}-0,08 x^{3}$ & 0,68 & 0,019 & $\downarrow / \uparrow / \downarrow$ \\
\hline Região Norte & $y=35,90+0,29 x$ & 0,16 & 0,246 & - \\
\hline Região Nordeste & $y=44,87+1,49 x$ & 0,89 & $<0,001$ & $\uparrow$ \\
\hline Região Sudeste & $y=101,24-0,55 x$ & 0,36 & 0,068 & - \\
\hline Região Sul & $y=159,16+0,13 x$ & 0,09 & 0,405 & - \\
\hline Região Centro-Oeste & $y=81,64+0,20 x$ & 0,07 & 0,471 & - \\
\hline
\end{tabular}

Doenças Cerebrovasculares

\begin{tabular}{lcccc}
\hline Brasil & $y=55,40-0,91 x+0,28 x^{2}$ & 0,77 & 0,002 & $\downarrow / \uparrow$ \\
\hline Região Norte & $y=39,43-0,63 x+0,27 x^{2}$ & 0,62 & 0,022 & $\downarrow$ \\
\hline Região Nordeste & $y=46,93+1,50 x+0,40 x^{2}-0,08 x^{3}$ & 0,91 & $<0,001$ & $\downarrow / \uparrow$ \\
\hline Região Sudeste & $y=60,36-1,23 x$ & 0,71 & 0,002 & $\downarrow$ \\
\hline Região Sul & $y=70,50-1,20 x+0,31 x^{2}$ & 0,95 & $<0,001$ & $\downarrow / \uparrow$ \\
\hline Região Centro-Oeste & $y=56,05-1,12 x$ & 0,51 & 0,02 & $\downarrow$
\end{tabular}

${ }^{\star} \downarrow$ Decrescente; $\uparrow$ Crescente; $\downarrow / \uparrow$ Decrescente/Crescente.

As internações por DIC apresentaram tendência de aumento, entretanto ao final do período analisado, ocorreu ligeira redução média de $-0,08$ ao ano $\left(r^{2}=0,68, p=0,019\right)$. A região Nordeste foi a única que demonstrou tendência crescente, com aumento médio anual de 1,49 $\left(r^{2}=0,89, p=<0,001\right)$, as demais apresentaram constância nas taxas de internação (Tabela 1).

Quanto às DCbV, identificou-se tendência decrescente na maior parte dos anos, com ligeiro aumento médio ao ano de 0,28 $\left(r^{2}=0,77, p=0,002\right)$ somente no final do período analisado, em todo território brasileiro. Nos aspectos regionais, Nordeste e Sul exibiram 
modelos de terceira e segunda ordem respectivamente, inicialmente com tendência decrescente e, posteriormente, crescente. O Sudeste apresentou redução significativa quando comparada com outras regiões, com média nas taxas de internação de 1,23 ao ano $\left(r^{2}=0,71, p=0,002\right)$ (Tabela 1$)$.

Na análise por grupos etários e por quadriênios, evidenciou-se o aumento exponencial no número de internações por DCV conforme o avançar da idade, sendo que os indivíduos na faixa etária entre 50 e 59 anos demonstraram a maior taxa de internações por todas as doenças (Figura 2).

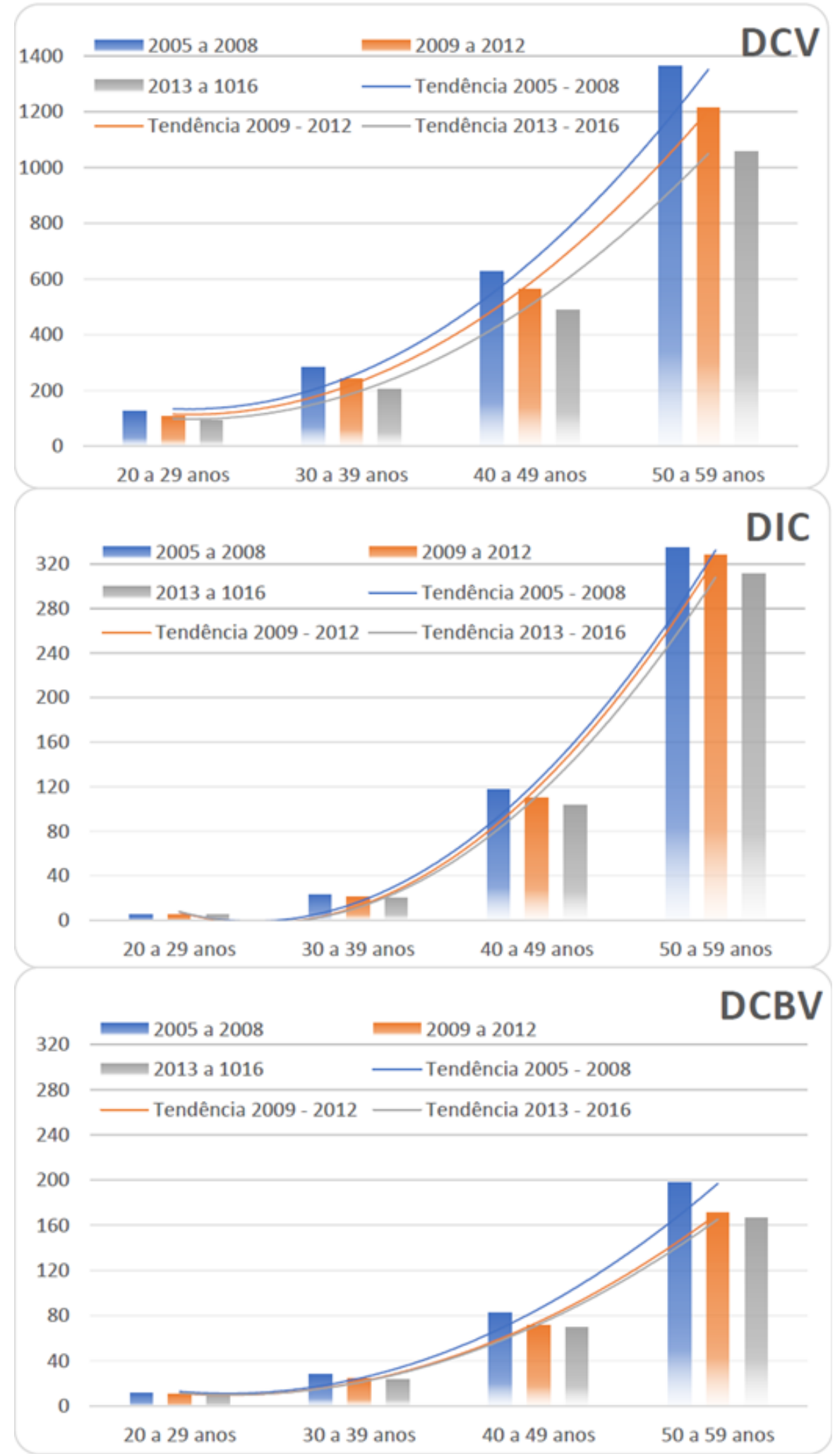

Figura 2 - Taxas de internações por doenças cardiovasculares em adultos, segundo faixa etária no Brasil, de 2005 a 2016. Maringá, PR, Brasil, 2017 
Observou-se que em ambos os sexos houve tendência decrescente nas taxas de internação por DCV em todas as regiões brasileiras, entretanto no sexo feminino notou-se uma redução mais expressiva (Brasil, $\left.-11,40, r^{2}=0,98, p=0,001\right)$. Quanto às DIC, identificaramse tendência decrescente/crescente/decrescente, nos três quadriênios analisados, para as internações em homens e mulheres no país. Constatou-se diferenças entre as regiões: Nordeste e Centro-Oeste apresentaram tendência crescente em ambos os sexos, com maior taxa média de internação entre os homens $(+2,23$ e $+1,50$, respectivamente). No que concerne às $\mathrm{DCbV}$, destaca-se a região Sudeste, cujas taxas de internação revelaramse decrescentes, sendo que para o sexo masculino a redução média ao ano foi de 1,75 $\left(r^{2}=0,73, p=0,002\right)$ e para o feminino de $0,73\left(r^{2}=0,64, p=0,005\right)$ (Tabela 2).

Tabela 2 - Análise da tendência das taxas de internação hospitalar de 2005 a 2016, por doenças cardiovasculares em adultos, segundo sexo. Maringá, PR, Brasil, 2017 (continua)

Local

Masculino

Feminino

\begin{tabular}{|c|c|c|c|c|c|c|c|c|}
\hline & Modelo & $\mathbf{R 2}$ & $\mathbf{p}$ & Tendência & Modelo & $\mathbf{R 2}$ & $\mathbf{p}$ & Tendência \\
\hline \multicolumn{9}{|c|}{ Doenças Cardiovasculares } \\
\hline Brasil & $y=431,68-9,46 x$ & 0,98 & $<0,001$ & $\downarrow$ & $y=452,54-11,40 x$ & 0,98 & $<0,001$ & $\downarrow$ \\
\hline Norte & $y=288,62-8,11 x$ & 0,96 & $<0,001$ & $\downarrow$ & $y=328,03-10,19 x$ & 0,71 & 0,002 & $\downarrow$ \\
\hline Nordeste & $y=324,51-2,18 x$ & 0,76 & 0,001 & $\downarrow$ & $y=364,90-8,32 x$ & 0,97 & $<0,001$ & $\downarrow$ \\
\hline Sudeste & $\begin{array}{c}y=467,78- \\
12,22 x\end{array}$ & 0,97 & $<0,001$ & $\downarrow$ & $y=466,16-9,90 x$ & 0,95 & $<0,001$ & $\downarrow$ \\
\hline Sul & $y=587,11-9,07 x$ & 0,98 & $<0,001$ & $\downarrow$ & $y=622,40-13,63 x$ & 0,98 & $<0,001$ & $\downarrow$ \\
\hline Centro-Oeste & $\begin{array}{c}y=441,26- \\
18,02 x\end{array}$ & 0,98 & $<0,001$ & $\downarrow$ & $y=477,87-24,52 x$ & 0,97 & $<0,001$ & $\downarrow$ \\
\hline
\end{tabular}

Doenças Isquêmicas do coração

\begin{tabular}{|c|c|c|c|c|c|c|c|c|}
\hline Brasil & $\begin{array}{c}y=113,09+1,53 x+ \\
0,17 x^{2}-0,09 x^{3}\end{array}$ & 0,7 & 0,016 & $\downarrow / \uparrow / \downarrow$ & $\begin{array}{c}y=62,25+0,79 x+ \\
0,15 x^{2}-0,07 x^{3}\end{array}$ & 0,75 & 0,01 & $\downarrow / \uparrow / \downarrow$ \\
\hline Norte & $\begin{array}{c}y=45,76 \\
+0,37 x+0,32 x^{2}\end{array}$ & 0,85 & 0,001 & $\downarrow / \uparrow$ & $y=22,99-0,11 x$ & 0,06 & 0,506 & - \\
\hline Nordeste & $y=55,71+2,23 x$ & 0,92 & $<0,001$ & $\uparrow$ & $y=34,42+0,78 x$ & 0,79 & 0,001 & $\uparrow$ \\
\hline Sudeste & $y=134,09-0,09 x$ & 0,44 & 0,038 & $\downarrow$ & $\begin{array}{l}y=67,86+0,76 x \\
+0,20 x^{2}-0,09 x^{3}\end{array}$ & 0,74 & 0,011 & $\downarrow / \uparrow / \downarrow$ \\
\hline Sul & $y=200,75+0,23 x$ & 0,18 & 0,218 & - & $y=118,10+0,01 x$ & 0,01 & 0,932 & - \\
\hline Centro-Oeste & $y=100,15+1,50 x$ & 0,76 & 0,001 & $\uparrow$ & $y=63,33-1,07 x$ & 0,68 & 0,003 & $\downarrow$ \\
\hline \multicolumn{9}{|c|}{ Doenças Cerebrovasculares } \\
\hline Brasil & $\begin{array}{c}y=58,58 \\
1,23 x+0,33 x^{2}\end{array}$ & 0,8 & 0,001 & $\downarrow / \uparrow$ & $\begin{array}{c}y=52,28- \\
0,60 x+0,23 x^{2}\end{array}$ & 0,71 & 0,005 & $\downarrow / \uparrow$ \\
\hline Norte & $\begin{array}{c}y=40,82- \\
0,77 x+0,30 x^{2}\end{array}$ & 0,68 & 0,007 & $\downarrow / \uparrow$ & $\begin{array}{l}y=37,38+0,71 x \\
+0,34 x^{2}-0,09 x^{3}\end{array}$ & 0,89 & 0,001 & $\downarrow / \uparrow / \downarrow$ \\
\hline Nordeste & $\begin{array}{c}y=49,25+0,66 x+ \\
0,26 x^{2}\end{array}$ & 0,76 & 0,003 & $\downarrow / \uparrow$ & $\begin{array}{l}y=45,21+1,32 x \\
+0,43 x^{2}-0,09 x^{3} \\
\end{array}$ & 0,9 & 0,001 & $\downarrow / \uparrow$ \\
\hline Sudeste & $y=65,45-1,75 x$ & 0,73 & 0,002 & $\downarrow$ & $y=55,40-0,73 x$ & 0,64 & 0,005 & $\downarrow$ \\
\hline Sul & $\begin{array}{c}y=74,12- \\
1,60 x+0,35 x^{2}\end{array}$ & 0,96 & $<0,001$ & $\downarrow / \uparrow$ & $\begin{array}{c}y=66,93- \\
0,79 x+0,25 x^{2}\end{array}$ & 0,9 & $<0,001$ & $\downarrow / \uparrow$ \\
\hline
\end{tabular}



Centro-Oeste
$y=55,60-$
0,83
0,001
$y=52,62-0,54 x$
0,33
0,08
$2,18 x+0,49 x^{2}$

$\uparrow$ Crescente; $\downarrow$ Decrescente; - Constante; $\uparrow / \downarrow$ Crescente/Decrescente; $\downarrow / \uparrow / \downarrow$ Decrescente/Crescente/Decrescente.

\section{DISCUSSÃO}

O estudo possibilitou analisar a tendência das taxas de internação por DCV em todo o território brasileiro no período de 12 anos, divididos em quadriênios. Os dados revelaram redução das hospitalizações em adultos, com variações entre as regiões, faixa etária e sexo. Oss achados são semelhantes ao encontrado pelo Estudo Global de Carga de Doenças, o qual identificou que, entre 1990 e 2013, a taxa de mortalidade por DCV diminuiu cerca de $22 \%$ e houve redução de um quinto da taxa de mortalidade por DIC e $A V E$, os quais podem impactar no número de hospitalizações ${ }^{(10)}$.

Contrariamente aos resultados detectados por este estudo, pesquisa conduzida entre 2001 e 2015, em uma região da Espanha, identificou tendência crescente nas hospitalizações por doenças cardiovasculares agudas, ao passo que, para a mortalidade, a tendência mostrou-se decrescente, enfatizando-se os efeitos das medidas de prevenção atribuídas ${ }^{(11)}$. Ainda, estudo transversal realizado nos Estados Unidos da América, com 40.082 internações por diagnóstico primário de acidente vascular cerebral isquêmico, entre 2010 e 2012, identificou que 41,5\% das hospitalizações ocorreram em sujeitos entre 18 e 54 anos, sendo que 43\% tiveram repetidas readmissões hospitalares e $25,6 \%$ dos casos ocorreram em associação a fibrilação atrial ${ }^{(12)}$.

No Brasil, estudo detectou que, entre os anos de 2010 e 2015, houve redução nas internações clínicas por DCV, entretanto, as hospitalizações cirúrgicas tiveram um aumento de $55 \%$, relacionadas principalmente a cirurgias vasculares e angioplastia coronariana ${ }^{(13)}$. Assim, em vista da magnitude e potencial de morbimortalidade que as doenças crônicas não transmissíveis (DCNT) representam, em especial as DCV, a Organização Mundial da Saúde (OMS) propôs uma redução de $25 \%$ das DCNT até o ano de 2025, e reforçou a importância da intervenção direta sobre a prevenção de fatores de risco mais prevalentes, com a necessidade de programas bem conduzidos e ações de prevenção primária e secundária para o diagnóstico e tratamento precoce das $\mathrm{DCV}^{(14)}$.

Em adesão a essa meta proposta, o Ministério da Saúde (MS), em parceria com outros ministérios e instituições, elaborou o Plano de Ações Estratégicas para o Enfrentamento das DCNT no Brasil, de 2011 a 2022, com o objetivo de criar e implantar políticas públicas efetivas direcionadas à prevenção e controle das DCNT, baseadas na vigilância, promoção da saúde e no cuidado integral, com o intuito de preparar o Brasil para o enfrentamento das DCNT ${ }^{(15)}$.

Pressupõe-se que a tendência de queda das internações no Brasil esteja relacionada a políticas que foram implementadas ao longo dos anos. Pode-se destacar a Política Nacional de Promoção da Saúde (PNPS), que reforça as obrigações de cada esfera do governo e a participação ativa do sujeito no cuidado à sua saúde na mobilização para alimentação saudável, prática de atividade física, enfrentamento do uso abusivo do álcool e abandono do tabaco(16).

Neste estudo, o Centro-Oeste foi a região que apresentou tendência decrescente das taxas de internação quando comparada com outras regiões brasileiras. Outro estudo, que analisou a tendência de internações por DCNT nos anos 2002 a 2012, demonstrou estabilidade para as taxas de internação por DCV no Brasil, para todas as regiões, exceto para a região Centro-Oeste, que foi a única que apresentou declínio significativo no 
período, com uma taxa de variação anual de $-8,78 \%{ }^{(17)}$. Em comparação a este estudo, as internações hospitalares por DCV apresentaram redução quando comparadas a anos anteriores, e a região Centro-Oeste se destaca em relação às demais.

Entretanto, as expressivas taxas de internação hospitalar por DCV, encontradas na região Sul, podem estar relacionadas ao maior número de leitos hospitalares, uma vez que esta região é detentora do maior índice de leitos por habitantes no Brasil(18). Outro estudo também mostrou maior prevalência de internações por DIC e DCbV na região Sul(3).

No Paraná, as regiões com melhores coberturas da Estratégia Saúde da Família (ESF) tiveram correlação com a redução no número de internações hospitalares por DCV. Ressalta-se que, atualmente, a região com melhor cobertura da ESF é a região Nordeste $(84,16 \%)$, seguida pela região Norte (68,59\%), Sul (68,36\%), Centro-Oeste (62,93\%) e Sudeste $(58,45 \%)^{(19)}$.

$\mathrm{Na}$ análise geral do presente estudo, observa-se que a região Sul possui a segunda melhor cobertura da ESF do país. Entretanto, foram encontradas as mais elevadas taxas de internação, destacando-se ainda que a região Centro-Oeste demonstrou maior redução das internações por DCV, e é a região com menor percentual de cobertura da ESF.

A maior prevalência de internações hospitalares para o sexo masculino corrobora resultados encontrados em estudo realizado no Nordeste da Espanha, em que a população masculina foi detentora de $70 \%$ das internações, principalmente por IAM (Infarto Agudo do Miocárdio) e com idade inferior a $46 \operatorname{anos}^{(20)}$.

No Brasil, estudo realizado na capital do Mato Grosso revelou que os homens tiveram maior probabilidade de apresentar dois ou mais fatores de risco para DCV e que esse aumento está diretamente associado à idade e menor escolaridade ${ }^{(21)}$. Outros estudos realizados no Brasil(22-23) também constataram que os homens têm maior possibilidade de apresentar dois ou mais fatores de risco para DCV. Na literatura internacional, estudo realizado na província de Jilin, na China $^{(24)}$ e entre os norte-americanos hispânicos/latinos de diferentes origens ${ }^{(20)}$, demonstraram o mesmo resultado. Contudo, em alguns estudos não foram encontradas diferenças significativas quanto ao número de fatores de risco cardiovascular por sexo ${ }^{(25-26)}$.

Portanto, embora tenha sido observada tendência geral de queda das DCV, a prevenção, redução e controle dessas doenças devem ser intensificados. A tarefa mais instigante e que pode ser atrelada à redução das DCV já é bem delimitada e consiste na redução dos fatores de risco na população, os quais podem ser mediados por meio da educação em saúde. Isso implica, sobretudo, na corresponsabilidade entre o indivíduo e os serviços de saúde com promoção da saúde, que, de certo modo, está relacionada ao direito de acesso à saúde de qualidade, a medidas preventivas efetivas que forneçam subsídios para o enfretamento de doenças já instaladas e prevenção de morbidades e agravos futuros.

Entre as limitações do estudo, salientamos que o SIH/SUS pode apresentar subnotificações em algumas localidades, devido à descentralização. Além disso, as AlHs são documentos que podem conter falhas na escrita e digitação, inclusive falhas no diagnóstico. Outra limitação é fato de o sistema contemplar apenas as internações financiadas pelo SUS, portanto, este estudo não abrange as internações dos sistemas particulares e convênios.

\section{CONCLUSÃO}

Observou-se tendência de declínio nas taxas de internações por DCV no Brasil, com variações quanto às $\mathrm{DIC}$ e $\mathrm{DCbV}$, e de acordo com o sexo. A região Centro-Oeste 
apresentou a maior redução anual média do período. Por outro lado, a região Sul do país apresentou as maiores taxas de internação para os três agrupamentos de doenças, o que sinaliza que novos estudos devem ser realizados a fim de identificar os fatores associados às altas taxas de internação hospitalar por doenças do aparelho circulatório nesta região.

Os resultados deste estudo podem repercutir nas ações de vigilância em saúde, ao favorecer a atuação do enfermeiro, o qual está presente em todos os níveis de atenção à saúde e atua de modo interdisciplinar, incentivando o autocuidado e a mudança dos fatores de riscos modificáveis por meio de estratégias de educação em saúde e psicoeducativas. Estas, por meio do desenvolvimento de coping, suscitam a promoção da saúde e prevenção de agravos, especialmente em regiões cujas taxas de internação apresentam-se elevadas, com tendência crescente e/ou possuem características que tornam a população vulnerável.

\section{AGRADECIMENTOS}

O presente trabalho foi realizado com apoio da Coordenação de Aperfeiçoamento de Pessoal de Nível Superior - Brasil (CAPES) - Código de Financiamento 001.

\section{REFERÊNCIAS}

1. World Health Organization (WHO). Noncommunicable diseases country profiles 2018 [Internet]. Geneva: World Health Organization; 2018 [acesso em 02 mar 2020]. Disponível em: https://www.who.int/ nmh/publications/ncd-profiles-2018/en/.

2. Cassiano MH, Silva HTD da, Luz ABS, Bezerra MS, Barbosa SS, Araújo DF de S. Correlation between anthropometric indices and blood pressure in adolescents and young adults in a municipality of northeast Brazil. Rev. Ciênc. Plur [Internet].2019 [acesso em 15 fev2020]; 5(2). Disponível em: https://periodicos.ufrn. br/rcp/article/view/18296/12009.

3. Bacurau AG de M, Ferraz R de O, Donalisio MR, Francisco PMSB. Mortalidade por doenças cerebrovasculares em idosos e a vacinação contra a influenza: estado de São Paulo, Brasil, 19802012. Cad. Saúde Pública[Internet]. 2019 [acesso em15 fev 2020]; 35(2). Disponível em: https://doi. org/10.1590/0102-311x00145117.

4. Gouveia M, Ascenção R, Fiorentino F, Costa J, Caldeira D, Broeiro-Gonçalves $P$, et al. The current and future burden of heart failure in Portugal. ESC Heart Failure [Internet]. 2019 [acesso em 05 fev 2020]; 6(2). Disponível em: https://doi.org/10.1002/ehf2.12399.

5. Park B, Park B, Han H, Choi EJ, Kim N, Shin Y, et al. Projection of the Years of Life Lost, Years Lived with Disability, and Disability-Adjusted Life Years in Korea for 2030. J Korean Med Sci [Internet].2019 [acesso em 05 fev 2020]; 34(suppl.1). Disponível em: https://doi.org/10.3346/jkms.2019.34.e92.

6. Massa KHC, Duarte YAO, Chiavegatto Filho ADP. Análise da prevalência de doenças cardiovasculares e fatores associados em idosos, 2000-2010. Ciênc. saúde coletiva [Internet]. 2019 [acesso em20 fev2020]; 24(1). Disponível em: https://doi.org/10.1590/1413-81232018241.02072017.

7. Ministério da Saúde (BR). Secretaria de Atenção à Saúde. Departamento de Regulação, Avaliação e Controle. Sistema de Informação Hospitalar - Manual Técnico Operacional do Sistema. Brasília: Ministério da Saúde; 2015.

8. Ministério da Saúde (BR). Sistema de Informações Hospitalares do SUS - SIH/SUS. Institucional 
[Internet]. [acesso em 19 out 2019]. Disponível em: http://tabnet.datasus.gov.br/cgi/deftohtm.exe?sih/ cnv/nruf.def.

9. Ministério da Saúde. Portaria n. 2.446, de 11 de novembro de 2014. Redefine a Política Nacional de Promoção da Saúde. Diário Oficial da República Federativa do Brasil, 13 nov 2014; Seção 1.

10. Roth GA, Abate D, Abate KH, Abay SM, Abbafati C, Abbasi N. et al. Global, regional, and national age-sex-specific mortality for 282 causes of death in 195 countries and territories, 1980-2017: a systematic analysis for the Global Burden of Disease Study 2017. The Lancet [Internet]. 2018 [acesso em03 fev 2020]; 392(10159). Disponível em: https://doi.org/10.1016/S0140-6736(18)32203-7.

11. Lopez-Messa JB, Llano JMA de, López-Fernandez L, Garcia-Cruces J, Garcia-Crespo J, Gonzales MP. Trends in hospitalization and mortality rates due to acute cardiovascular disease in Castile and Laón, 2001 to 2015. Rev Esp Cardiol [Internet]. 2017 [acesso em15 dez2019]; 17(2). Disponível em: http://dx.doi. org/10.1016/j.rec.2017.04.033.

12. Wang G, Joo H, Tong X, George MG. Hospital Costs Associated with Atrial Fibrillation for Patients with Ischemic Stroke Aged 18-64 Years in the United States. Stroke [Internet]. 2015 [acesso em 10 jan 2020]; 46. Disponível em: https://www.ahajournals.org/doi/full/10.1161/STROKEAHA.114.008563.

13. Siqueira A de SE, Siqueira-Filho AG de, Poirot MG. Analysis of the economic impact of cardiovascular diseases in the last five years in Brazil. Arq. Bras. Cardiol [Internet]. 2017 [acesso em 08 dez 2019]; 109(1). [Internet]. Disponível em: http://dx.doi.org/10.5935/abc.20170068.

14. Rocha RM, Martins W de A. Manual de prevenção cardiovascular[Internet]. São Paulo: Planmark; Rio de Janeiro: SOCERJ - Sociedade de Cardiologia do Estado do Rio de Janeiro; 2017 [acesso em 27 nov2019]. Disponível em: https://socerj.org.br/antigo/wp-content/uploads//2017/05/Manual de Prevencao Cardiovascular SOCERJ.pdf.

15. Câmara C. Mapeamento político da saúde no Brasil com foco em diabetes e doenças cardiovasculares[Internet]. São Paulo: Sociedade Brasileira de Diabetes; 2018 [acesso em 27 nov2019]. Disponível em: https://www.diabetes.org.br/publico/images/mapeamento-diabetes.pdf.

16. Dias MS de A, Oliveira IP de, Silva LMS da, Vasconcelos MIO, Machado M de FAS, Forte FDS, Silva LCC da. Política Nacional de Promoção da Saúde: um estudo de avaliabilidade em uma região de saúde no Brasil. Cienc. saúde coletiva [Internet]. 2018 [acesso em 10 jan 2020]; 23(1). Disponível em: http:// dx.doi.org/10.1590/1413-81232018231.24682015.

17. Santos MAS, Oliveira MM de, Andrade SSC de A, Nunes ML, Malta DC, Moura L de. Noncommunicable hospital morbidity trends in Brazil, 2002-2012. Epidemiol Serv Saúde [Internet]. 2015 [acesso em 03 fev 2020]; 24(3). Disponível em: http://dx.doi.org/10.5123/S1679-49742015000300005.

18. Lins JGMG.A concentração de leitos nos municípios do Brasil pode estar associada a pressões de uso no sistema hospitalar do país? Braz. Ap. Sci. Rev [Internet]. 2019 [acesso em02 mar 2020];3(4).Disponível em: https://doi.org/10.34115/basrv3n4-002.

19. Ministério da Saúde (BR). Departamento de Atenção Básica - DAB. Secretaria de Atenção à Saúde. Informação e Gestão da Atenção Básica. Cobertura da atenção básica. [Internet]. 2017 [acesso em 05 mar 2020]. Disponível em: https://egestorab.saude.gov.br/paginas/acessoPublico/relatorios/ relHistoricoCoberturaAB.xhtml.

20. Ding D, Kolbe-Alexander T, Nguyen B, Katzmarzyk PT, Pratt M, Lawson KD. The economic burden of physical inactivity: a systematic review and critical appraisal. Br J Sports Med [Internet]. 2017 [acesso em 03 mar 2020];51. Disponível em: http://doi.org/10.1136/bjsports-2016-097385.

21. Boiça LG de O, Martins MSAS, Segri NJ, Guimarães LV, Durante GD, Malta D de C. Simultaneidade de fatores de risco cardiovascular e fatores associados em adultos. Connection Line [Internet]. 2018 [acesso em28 jan2020]; 19. Disponível em: http://dx.doi.org/10.18312\%2F1980-7341.n19.2018.1197.

22. Duarte APP, Rodrigues PRM, Ferreira MG, Cunha DB, Moreira NF, Sichieri, et al. Socio-economic and demographic characteristics associated with risk behaviour patterns for chronic non-communicable 
diseases in Brazil: data from the National Health Survey, 2013. Public Health Nutrition [Internet].2019 [acesso em12 mar 2020]; 22(11). Disponível em: https://doi.org/10.1017/S136898001900034X.

23. Gonçalves RPF, Haikal DS, Freitas MI de F, Machado ÍE, Malta DC. Self-reported medical diagnosis of heart disease and associated risk factors: National Health Survey. Rev. bras. epidemiol. [Internet]. 2019 [acesso em28 jan 2020]; 22(supl.2). Disponível em: https://doi.org/10.1590/1980-549720190016.supl.2.

24. Yu J, Ma Y, Yang S, Pang K, Yu Y, Tao Y, et al. Risk factors for cardiovascular disease and their clustering among adults in jilin (China). Int J Environ Res Public Health [Internet]. 2016 [acesso em 10 dez 2019];13(1).Disponível em: https://doi.org/10.3390/ijerph13010070.

25. Quintela SHC, Siqueira PG, Höffling FB, Imbriani BH, Romero M de V, Silva RM da. The perceptions of vascular dysfunction bearing patients with regards to health education as an autonomy instrument. Rev Fun Care Online [Internet]. 2019 [acesso em28 jan2020]; 11(1). Disponível em: http://www.seer.unirio.br/ index.php/cuidadofundamental/article/view/6443.

26. Medeiros PA de, Cembranel F, Figueiró TH, Souza BB de, Antes DL, Silva DAS, et al. Prevalence and simultaneity of cardiovascular risk factors in elderly participants of a population-based study in southern Brazil. Rev Bras Epidemiol [Internet]. 2019 [acesso em 03 fev 2020]; 22(e190064). Disponível em: https:// doi.org/10.1590/1980-549720190064. 
COMO REFERENCIAR ESTE ARTIGO:

Figueiredo FSF, Rodrigues TFC da S, Cardoso LCB, Santos FGT dos, Oliveira RR de, Radovanovic CAT. Declínio das taxas de internação hospitalar por doenças cardiovasculares em adultos no Brasil. Cogitare enferm. [Internet]. 2021 [acesso em "colocar data de acesso, dia, mês abreviado e ano"]; 26. Disponível em: http://dx.doi. org/10.5380/ce.v26i0.72327.

*Artigo extraído da dissertação de mestrado "Análise das internações hospitalares por doenças cardiovasculares em adultos no Brasil". Universidade Estadual de Maringá, 2018.

Recebido em: 21/03/2020

Aprovado em: 24/07/2020

Autor Correspondente:

Luana Cristina Bellini Cardoso

Universidade Estadual de Maringá - Maringá, PR, Brasil

E-mail: luana.bellini@hotmail.com

Contribuição dos autores:

Contribuições substanciais para a concepção ou desenho do estudo; ou a aquisição, análise ou interpretação de dados do estudo - FSFF, TFCSR, LCBC, FGTS, TTO, CATR

Elaboração e revisão crítica do conteúdo intelectual do estudo - FSFF, TFCSR, LCBC, FGTS, TTO, CATR

Aprovação da versão final do estudo a ser publicado - FSFF, TFCSR, LCBC, FGTS, TTO, CATR

Copyright () 2021 Este é um artigo em acesso aberto distribuído nos termos da Licença Creative Commons Atribuição, que permite o uso irrestrito, a distribuição e reprodução em qualquer meio desde que o artigo original seja devidamente citado. 